DOI: https://doi.org/10.46296/gt.v4i8edesp.0031

\title{
NIVELES DE ANSIEDAD EN LOS ANCIANOS DURANTE LA CRISIS SANITARIA DEL COVID-19
}

\section{ANXIETY LEVELS IN THE ELDERLY DURING THE SANITARY COVID-19 CRISIS}

\author{
Mera-Posligua Mauro Julio ${ }^{\text {; }}$; Márquez-Fernández Luis ${ }^{2}$ \\ ${ }^{1}$ Estudiante de la Maestría Académica con Trayectoria de Investigación en Psicología, Mención \\ Psicoterapia. Instituto de Posgrado de la Universidad Técnica de Manabí, UTM. Portoviejo, \\ Ecuador. Correo: mjmera95@gmail.com. ORCID ID: https://orcid.org/0000-0001-9560-6395 \\ 2 Docente Investigador de la Universidad Técnica de Manabí, UTM. Portoviejo, Ecuador. \\ Correo: luis.marquez01@utm.edu.ec. ORCID ID: https://orcid.org/0000-0003-3318-3416
}

\begin{abstract}
Resumen
La ansiedad es una reacción fisiológica y psíquica que altera el estado mental al responder a estímulos externos o internos, manifestando intranquilidad, incertidumbre y excitación. Esta cumple un rol adaptativo en el ser humano, no obstante, cuando la reacción del individuo se desborda y no es proporcional a la intensidad del estímulo, se convierte en un trastorno psicológico. El presente artículo tiene como finalidad inferir los niveles de ansiedad presentes en la población de ancianos ecuatoriana, durante la crisis sanitaria del COVID-19 causada por el virus del SARS CoV -2. El método utilizado para validar la investigación es el análisis estadístico descriptivo. Se identificaron los niveles de ansiedad presentes en los jubilados de la mancomunidad de Manta, Montecristi y Jaramijó durante esta pandemia mundial. Se utilizó el inventario de ansiedad de Beck para la detección de los niveles de ansiedad, el cual se fundamentó en un estudio de campo realizado de manera virtual. Los resultados reflejaron niveles de ansiedad leve y un considerable porcentaje de ansiedad moderada y grave. Por otra parte, se observó una mayor afectación en los niveles de ansiedad en el sexo femenino respecto a su opuesto.
\end{abstract}

Palabras clave: Ansiedad, pandemia, ancianos, vulnerabilidad.

\begin{abstract}
Anxiety is a physiological and psychic reaction that alters the mental state when responding to external or internal stimuli, manifesting restlessness, uncertainty and excitement. This fulfills an adaptive role in human beings. However, when the individual's reaction overflows and is not proportional to the intensity of the stimulus, it becomes a psychological disorder. The purpose of this article is to determine the levels of anxiety present in the Ecuadorian elderly population, during the health crisis of COVID-19 caused by the SARS CoV-2 virus. The method used to validate the research is a descriptive statistical analysis. The levels of anxiety present in pensioners from the Manta, Montecristi, and Jaramijo commonwealth during this global pandemic, were identified using the Beck anxiety inventory, which was based on a virtual field study. The results reflected mild levels of anxiety, and a considerable percentage of moderate and severe anxiety. On the other hand, there is a greater impact on anxiety levels in females, compared to males.
\end{abstract}

Keywords: anxiety, pandemic, elderly, vulnerability.

Información del manuscrito:

Fecha de recepción: 13 de julio de 2021.

Fecha de aceptación: 27 de septiembre de 2021.

Fecha de publicación: 12 de noviembre de 2021. 


\section{Introducción}

En los últimos meses del año 2019 se detectó una enfermedad denominada COVID-19, producida por el virus del SARS CoV-2. Fue declarada como pandemia por la Organización mundial de la salud OMS (2020), debido a su propagación por todo el mundo. Esto originó diversos cambios abruptos en el estilo de vida de la población.

Bosch (2012) comenta que "las personas y los grupos pueden hacerse vulnerables si se modifican sus condiciones de vida por cambios forzados en su entorno", lo cual hace alusión a los actuales momentos que vive la sociedad bajo el contexto de la pandemia producida por el virus del SARS-CoV-2.

En el estudio realizado por Gonzales A. y colaboradores (2020), midieron los niveles de ansiedad a causa del aislamiento social obligatorio producido por el virus del SARSCoV-2, en esta investigación el $20.9 \%$ de la población presentó ansiedad leve, el $13.4 \%$ ansiedad moderada, y el $9.0 \%$ presentó ansiedad severa.

Para Cabeza A. y colaboradores (2018), la ansiedad "es una respuesta del organismo a una alteración del estado mental, causada por un estímulo endógeno o exógeno que eleva los niveles de estrés y ansiedad", este tipo de ansiedad suele cumplir una función adaptativa.

En el estudio de Cedeño N. y colaboradores (2020), se menciona que el miedo y la ansiedad son necesarios para enfocarse en situaciones de peligro; la incertidumbre nos impulsa a mantener el control, evaluar posibilidades y evaluar los recursos con los que se cuentan; siendo la preocupación, un proceso cognitivo y emocional que anticipa las acciones a tomar frente al peligro.

La ansiedad es un fenómeno multicausal, debido a que puede producirse por una tendencia genética, traumas a lo largo del desarrollo vital, desbalances químicos en el cuerpo y como efecto colateral de alguna enfermedad o accidente físico (Rodríguez et al, 2008). La ansiedad es un elemento psicológico presente en todos los estratos sociales y contextos posibles, al mantenerse en niveles estables es favorable para las personas debido a que provee una 
descarga energética suficiente para afrontar obstáculos del diario vivir.

Se considera un problema cuando la ansiedad común se convierte en un trastorno de ansiedad, este es uno de los principales trastornos mentales, que, al no ser abordados tempranamente, suele generar dificultades en la funcionalidad de las personas, promoviendo vulnerabilidad ante otros cuadros sintomatológicos mentales de mayor complejidad.

Según Ramírez y colaboradores (2020) las complicaciones psicológicas pueden brotar de la falta de certeza del resultado final o de la ausencia de conocimiento respecto al tiempo de duración de la crisis sanitaria. También la posibilidad de que los planes a futuro cambien de forma dramática y la separación brusca del contexto social o familiar de las personas, son catalizadores frecuentes de cuadros de ansiedad.

Hernández J., (2020), indica que los grupos vulnerables, en este caso los ancianos, tienen una mayor probabilidad de que el actual contexto afecte de manera significativa su salud mental, debido a la paranoia que genera el confundir síntomas habituales de la edad con los del COVID-19, o no tener claro la forma de transmisión exacta de la enfermedad, ya que es un virus que se sigue estudiando, además de los efectos del distanciamiento social, aislamiento y efectos de cuarentena.

Los trastornos de ansiedad son perturbaciones psiquiátricas que se definen por el miedo excesivo a escenarios que no representan un peligro auténtico, los cuales terminan desgastando los recursos de las personas y generando otras enfermedades de carácter psicológico (Malllen et al, 2011).

La Organización Panamericana de la Salud (OPS) (2018), postula que los trastornos mentales en la Región de las Américas originan más de un tercio de la discapacidad total en las Américas, teniendo los trastornos de ansiedad una prevalencia entre 9,3 $\%$ y $16,1 \%$; porcentajes que afectan en mayor medida a los grupos vulnerables.

Varios autores han medido los niveles de ansiedad y otros factores relacionados durante esta pandemia. Así, Etxebarria y colaboradores (2020), determinaron niveles de ansiedad de moderados a 
graves en un $28 \%$ del total de la población en contraste con la investigación de Callís (2021) que los niveles de ansiedad variaban de leves a moderados y con una mayor afectación en adultos mayores de sexo femenino.

En un estudio similar Oblitas A., \& Sánchez L., (2020) realizada para verificar los niveles de ansiedad en adultos durante la pandemia del COVID-19, concluyó con respecto a la población femenina que el $29.6 \%$ presentaba ansiedad leve, el $11.1 \%$ ansiedad moderada y el $3.7 \%$ ansiedad severa; a diferencia de la población masculina, en la cual, el $15.0 \%$ presentó ansiedad leve, el 15.0\% presentó ansiedad moderada $y$ el $12.5 \%$ niveles de ansiedad severa, ambos sexos con edades entre 22 y 60 años.

Como lo expresa Ortiz J. et al (2018), la población que cruza por un periodo de aislamiento social, con restricción de movilidad, sumado al limitado contacto con otros, son vulnerables a presentar problemas psiquiátricos, desde síntomas aislados hasta el insomnio, ansiedad, depresión y trastorno por estrés postraumático; condiciones aproximadas a las que se viven en la actual crisis sanitaria.

El objetivo de este artículo es conocer los niveles de ansiedad presentes en la población de ancianos provenientes de la Unión de Asociaciones de Jubilados de la Mancomunidad de Manta, Montecristi y Jaramijó durante la pandemia provocada por el virus del SARS CoV-2, para concientizar sobre la vulnerabilidad de dicha población y generar nuevos datos estadísticos que sirvan de referencia a futuras investigaciones en torno a la prevención y tratamiento de trastornos mentales en la población de ancianos.

Se prevé que la población de ancianos provenientes de la unión de asociaciones de jubilados de la mancomunidad de Manta, Montecristi y Jaramijó, que experimentaron las medidas de confinamiento, presente niveles significativos de ansiedad durante la pandemia provocada por el virus del SARS CoV-2, lo cual podría complicar el desarrollo saludable durante esta etapa del ciclo vital, afectando la salud mental de esta población y reduciendo su calidad de vida. 


\section{Materiales y métodos}

\subsection{Diseño}

El tipo de investigación aplicado es estadístico descriptivo.

\subsection{Estrategia de búsqueda}

Los participantes fueron elegidos por el método de azar simple, con una muestra de 50 ancianos provenientes de la unión de asociaciones de jubilados de la mancomunidad de Manta, Montecristi y Jaramijó.

\subsection{Criterios de exclusión e inclusión}

El criterio de inclusión consistió en tener que estar registrados formalmente en dicha asociación. Como criterios de exclusión, que no contaran con un dispositivo móvil que permitiera realizar vídeo llamadas y sin acceso a internet ilimitado; y como criterio de eliminación, se descartarían las personas que desarrollaran afectaciones de salud durante el estudio que impidieran su participación en la investigación.

\subsection{Extracción de datos}

El instrumento utilizado fue el inventario de ansiedad de Beck en su versión española, cuyas propiedades psicométricas, fiabilidad y validez, resultan adecuadas para detectar y cuantificar síntomas de ansiedad en pacientes diagnosticados con trastornos psicológicos como se aprecia en la investigación de Sanz J., (2012).

\subsection{Análisis de datos}

Se solicitó el consentimiento informado, el cual fue emitido por el presidente de dicha asociación. Para el manejo correspondiente de datos estadísticos recabados se utilizó el software de Statistical Package for the Social Sciences (SPSS).

\section{Análisis de resultados}

El estudio se llevó a cabo con 50 ancianos pertenecientes a la unión de asociaciones de jubilados de la mancomunidad de las ciudades de Manta, Montecristi y Jaramijó, de la provincia de Manabí, cuyas edades estaban comprendidas entre los 60 y 80 años. Se aplicó la escala de ansiedad de Beck, cuyos resultados, como se puede apreciar en la tabla 1 , enuncian que el $84 \%$ de los ancianos evaluados presentaron niveles de ansiedad bajos, reflejando que el contexto de la pandemia 
influye mayormente de forma leve en los niveles de ansiedad dentro de esta población. En este nivel bajo, existe un mayor porcentaje de hombres (96\%), y en menor disposición las mujeres (76\%). Por otro lado, el $12 \%$ de nuestra muestra presentó niveles de ansiedad moderados. Dentro de este grupo se observa una mayor afectación en las mujeres, concretamente el doble. E 4\% restante presentó ansiedad grave, representado exclusivamente por mujeres.

Tabla 1. Grado de ansiedad en relación al sexo

Tabla cruzada de niveles de ansiedad según el sexo

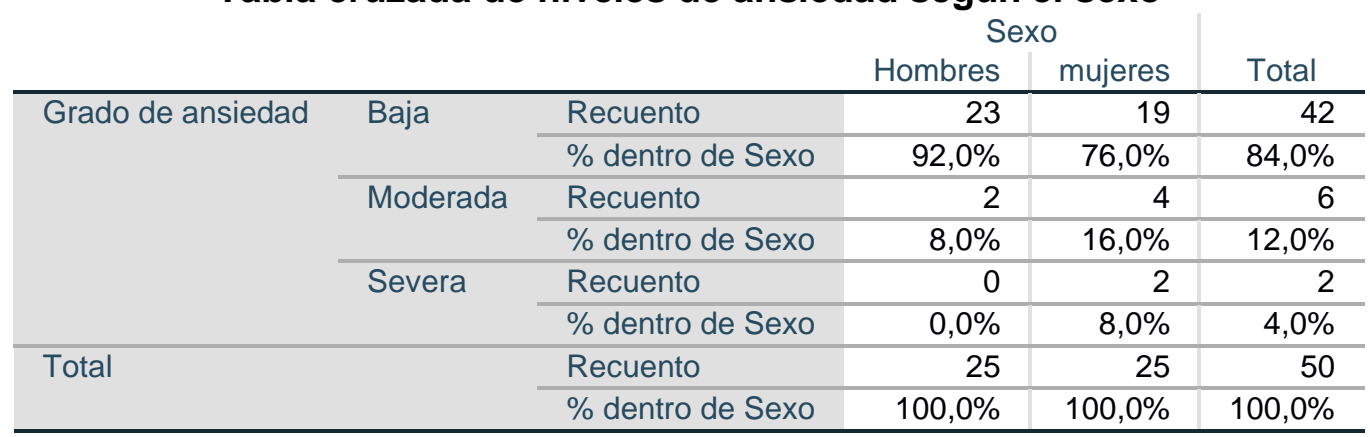

Como se puede apreciar en la tabla 2, el nivel de ansiedad bajo está presente en un porcentaje similar entre las edades de 60 a 71 años y ocupa en su totalidad la población de adultos mayores entre 72 y 85 años de edad. Los niveles de ansiedad moderados están presentes en porcentajes parecidos entre los rangos de 60 a 65, y de 66 a 71 años, mientras que la ansiedad severa solo es encontrada en el grupo de 60 a 65 años, lo cual es un indicador de que este grupo de edades sufren una mayor afectación durante la pandemia del COVID-19.

Tabla 2. Grado de ansiedad en relación a la edad.

Tabla cruzada de niveles de ansiedad según la edad

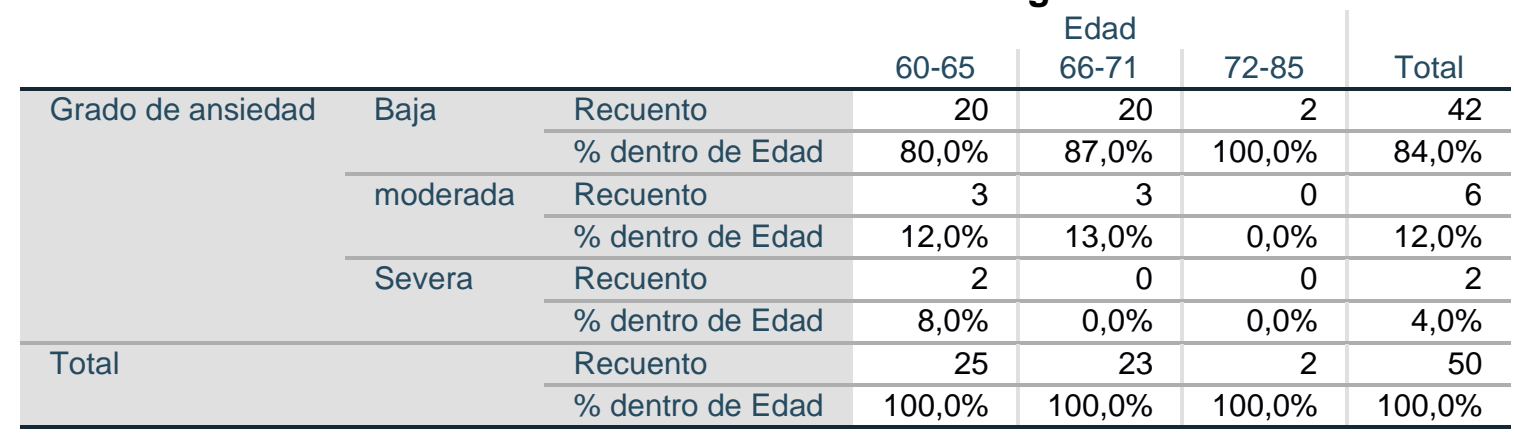


En el gráfico número uno, se observan las dos sintomatologías de mayor presencia en la población de adultos mayores, siendo el noveno ítem del inventario de ansiedad de Beck, "atemorizado o asustado", el de mayor presencia en dicha población durante la pandemia del COVID-19, con una afectación severa.

El segundo ítem con el que resonaron más los participantes fue el quinto, "con temor a que ocurra lo peor", debido a que representa el porcentaje más alto de afectación moderada en esta población, lo cual tiene mucho sentido, teniendo en cuenta que, en esta crisis, la paranoia, el distanciamiento social, el aislamiento, así como otros efectos o hechos indeseados de la cuarentena, han sido una vivencia muy extendida, debido a las condiciones específicas de esta crisis sanitaria. Donde los efectos del virus son muy desconocidos, y los medios de comunicación y redes sociales han generado un gran impacto emocional, incluso confusión, con las noticias difundidas al respecto.

Gráfico 1. Sintomatologías de mayor frecuencia.

9 Atemorizado o asustado.

50 respuestas
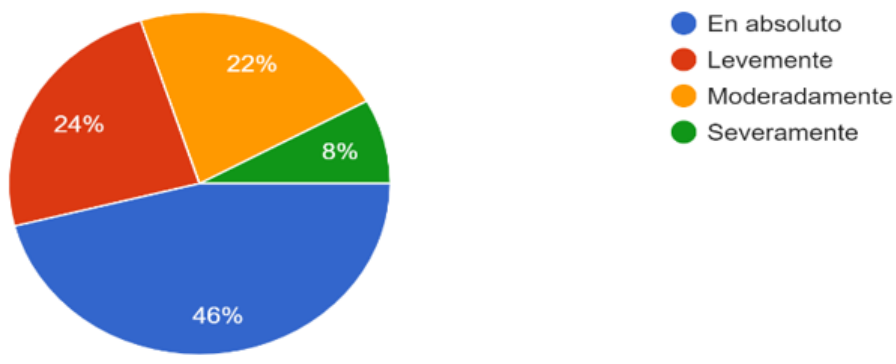

5 Con temor a que ocurra lo peor.

50 respuestas

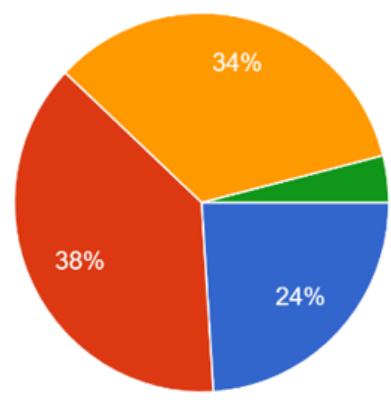

En absoluto

Levemente

Moderadamente

Severamente 
4. Discusión

Al determinar los niveles de ansiedad presentes en la población de ancianos pertenecientes a la asociación de jubilados de la mancomunidad de las ciudades de Manta, Montecristi y Jaramijó durante la pandemia producida por el virus del SARS COV-2, se observa una alteración significativa en los niveles de ansiedad de los ancianos. Donde el sexo femenino presenta una mayor intensidad en el grado de afectación de la ansiedad en relación al sexo masculino.

Estos resultados apuntan a que las mujeres han sufrido una mayor afectación en los cuadros de ansiedad durante la pandemia, tal como sucedió en la investigación de Callís S., (2021). Se hace necesario una intervención multidisciplinaria para minimizar el riesgo a desarrollar patologías de mayor complejidad.

En la investigación de Flores M., et al (2012), se aprecian resultados similares al existir una mayor afectación en el sexo femenino. Tomando en cuenta que este es un estudio realizado en ancianos, años antes de la pandemia del COVID-19; se confirma que, a pesar de los cambios ejercidos en la vida de los ancianos por el actual contexto, la afectación en los niveles de ansiedad tiende a ser más elevados en la población de sexo femenino, existiendo un mayor porcentaje en los niveles de ansiedad moderados y graves.

Llama la atención los resultados obtenidos en la investigación de Callís (2021), la cual se llevó a cabo en abril y mayo de 2020 en Cuba, durante la pandemia del COVID-19, en una población conformada exclusivamente por ancianos, a pesar de las diferencias de edades y lugar de residencia, se siguen manteniendo resultados similares a la investigación realizada por Flores M., et al (2012) donde el sexo femenino presentaba una mayor afectación en los niveles de ansiedad cognitiva en relación a los hombres.

Resultados similares también se pueden apreciar en el estudio de Oblitas A., y Sánchez L., (2020), desarrollado en Perú durante una parte del período de aislamiento social obligatorio por COVID-19. Concretamente, desde el 16 de marzo al 30 de junio de 2020, y el cual incluía a 67 adultos de entre 22 y 60 años de edad. En este estudio 
se observa que, la afectación en los niveles de ansiedad sigue siendo acusada en mayor medida por la población de sexo femenino, a pesar de ser una muestra con una gran diversidad de edades.

Es de considerar, que el foco de la presente investigación se ha centrado en la medición de los niveles de ansiedad de una muestra de ancianos en el transcurso de la pandemia del COVID-19, pero no se han registrado otras variables, ni su interacción entre ellas o con los propios niveles de ansiedad. Nos referimos a factores como posible asistencia terapéutica por parte de alguna entidad, comorbilidades, características sociodemográficas, atribución subjetiva de estos niveles a la pandemia, hábitos y estilo de vida... Y lo que es más importante, medidas de ansiedad antes de que estallara la pandemia. Por ello, no se puede establecer una relación directa y estadísticamente significativa entre el contexto del Covid-19 y el incremento de los niveles de ansiedad, pero sí un acercamiento a lo postulado por Bosch (2012) el cual menciona que las personas aumentan su vulnerabilidad en situaciones de cambios forzados, como los generados a raíz de la pandemia por el COVID-19.

Por su parte, Ramírez (2020) menciona que las complicaciones psicológicas pueden aparecer durante la pandemia del COVID-19, causado por la falta de certeza del resultado final, tiempo de duración de crisis sanitaria, cambio de los planes a futuro y la separación brusca del contexto social o familiar; elementos catalizadores de los cuadros de ansiedad. Hernández (2020) también recalca esta posibilidad, afirmando que el actual contexto afecta a la salud mental, y en mayor medida a la población vulnerable, como lo son los ancianos. Todo lo cual sugiere una más que hipotética relación entre el actual contexto y las afectaciones en los niveles de ansiedad.

\section{Conclusiones}

La investigación realizada concluye, que existe una afectación moderada de los niveles de ansiedad en los ancianos pertenecientes a la asociación de jubilados de la mancomunidad de las ciudades de Manta, Montecristi y Jaramijó. 
Siendo la población femenina quien muestra una mayor afectación.

Esto concuerda con los estudios de vulnerabilidad en relación a los estudios sobre trastornos de ansiedad realizados por Arenas (2009), quien, basado en un enfoque psicobiológico, afirma que el sexo femenino tiene una mayor disposición a generar afectaciones en los niveles de ansiedad; lo cual ha sido observado tanto en la adultez como en la vejez. Dicha perspectiva tendría sentido que se mantuviese o agravase durante un momento de alto impacto emocional, como lo ha sido el contexto que ha acompañado a la irrupción del COVID-19 a nivel mundial.

El grupo de edad más afectado es el de 60 a 71 años, siendo el único en presentar niveles graves de ansiedad, lo cual podría deberse a que muchas de estas personas aun estando jubiladas, mantienen contacto con responsabilidades en la sociedad, las cuales se han dificultado notablemente debido a los cambios y medidas impuestas durante esta crisis sanitaria.

Los síntomas de mayor afectación encontrados en el cuestionario de ansiedad de Beck son: el estar atemorizado y la sensación de que va a ocurrir lo peor, pareciendo que son estos síntomas más psíquicos y relacionados con lo subjetivo y la falta de concreción, los que ha generado la expansión de este virus.

Los ancianos son una población vulnerable per se, los cuales se han visto obligados a adaptarse a un nuevo contexto, el cual trae consigo una mayor desconexión social, noticias de alto impacto emocional y restricción de las pocas actividades de autonomía y entretenimiento de las que gozaban. Al margen de esto, han debido lidiar con la idea de que tienen una de las tasas de mortalidad más altas por esta enfermedad.

A pesar de que los niveles de ansiedad graves y moderados, se presentan en menor medida que los niveles de ansiedad leve, los ancianos que cruzan por un periodo de aislamiento social, con restricciones de movilidad, sumado al limitado contacto con otros, podrían incrementar los niveles de ansiedad actuales, dando paso a otras afectaciones de mayor complejidad en su salud mental (Ortiz J. et al., 2018).

Esto nos lleva a reflexionar sobre la importancia de ofrecer a esta 
población una mejor calidad de vida

a través de la creación de programas

multidisciplinarios que les permita mantener por más tiempo el mejor estado posible de salud física y mental. Programas accesibles y que cumplan un rol preventivo en el desarrollo de enfermedades de mayor complejidad en esta población tan vulnerable.

\section{Bibliografía}

Bosch Alberto., (2012). Resiliencia. Revista educación médica. 15 (2).

http://scielo.isciii.es/scielo.ph p?script=sci_arttext\&pid=s15 75-18132012000200004

Bonanno George., Kaltman Stacey., (2001). las variedades de experiencias de duelo. Clinical Psychology Review. 21 https://doi.org/10.1016/s02727358(00)00062-3

Carretero Raúl. (2010). Resiliencia. una visión positiva para la prevención e intervención desde los servicios sociales. Revista. Nómadas. 27 (3). https://www.redalyc.org/pdf/1 81/18113757004.pdf

Callís Sureima., Guarton Omara., Cruz Virgen., Armas Adam., (2021) Estados afectivos emocionales en adultos mayores solos en aislamiento social durante la pandemia
COVID-19. Revista Dr. Zoilo E. Marinello Vidaurreta. 46 (1).

http://revzoilomarinello.sld.cu/ index.php/zmv/article/view/24 91/pdf_739

Cedeño Nereida., Vélez María., Duran Ányelo., Torres Mariana., (2020). Afrontamiento del covid-19: estrés, miedo, ansiedad y depresión.

Revista.

Enfermería investiga. 5 (32020).

https://revistas.uta.edu.ec/ere vista/index.php/enfi/article/vie w/913/858

Comité de operaciones de emergencia nacional. (2020). informe de situación covid-19 ecuador.

https://www.gestionderiesgos. gob.ec/wpcontent/uploads/2020/03/infor me-de-situaci\%c3\%b3nno008-casos-coronavirusecuador-16032020-20h00.pdf

Etxebarria Naiara., Santamaria María., Gorrochategui Maitane., Mondragon Nahia., (2020). Niveles de estrés, ansiedad y depresión en la primera fase del brote del covid-19 en una muestra recogida en el norte de españa. Revista. Cadernos de Saúde Pública. $36 \quad$ (1). https://doi.org/10.1590/0102$311 \times 00054020$

Flores María., Cervantes Guillermo., González Guillermo., Vega María., Barbosa María., 
(2012) Ansiedad y depresión como indicadores de calidad de vida en adultos mayores. https://dialnet.unirioja.es/desc arga/articulo/5154971.pdf

Gonzales Anibal., Sánchez Nanci., (2020). Ansiedad en tiempos de aislamiento social por covid-19. Revista. Av Enferm. 38

https://revistas.unal.edu.co/in dex.php/avenferm/article/view /87589/76025

Hernández José., (2020) Impact of COVID-19 on people's mental health. Revista Medicentro Electrónica $24 \quad$ (3). http://scielo.sld.cu/scielo.php? script=sci_arttext\&pid=S1029 $-30432020000300578$

Sanz Jesús., Vera María., Fortún María., (2012). El "inventario de ansiedad de Beck" (bai): propiedades psicométricas de la versión española en pacientes con trastornos psicológicos. Revista Behavioral Psychology 20(3). https://www.behavioralpsycho .com/wpcontent/uploads/2019 /08/05.Sanz_20-3oa.pdf

Mallen María., Garza David., (2011). Trastornos de ansiedad. Revista. Neurología, Neurocirugía y Psiquiatría. 44 (3).

https://www.medigraphic.com /pdfs/revneuneupsi/nnp2011/nnp113d.pdf

Oblitas Anibal., Sánchez Nancy., (2020) Ansiedad en tiempos de aislamiento social por COVID-19. Chota, Perú. https://revistas.unal.edu.co/in dex.php/avenferm/article/view /87589/76025

Ortiz Jairo., Quintero Diego., Córdoba Carmen., Ceballos Francisco., \& Córdoba Franklin., (2018). Consecuencias de la pandemia covid 19 en la salud mental asociadas al aislamiento social. Revista. Cubana de Investigaciones Biomédicas. 37 (4). http://www.scielo.org.co/pdf/r $\mathrm{ca} / \mathrm{v} 48 \mathrm{n} 4 / \mathrm{es}$ 2256-2087-rca48-04-e301.pdf

OMS. (2017). La salud mental en el lugar de trabajo. https://www.who.int/mental_h ealth/es/\#: :text=m\%c3\%a1s $\% 20$ de\%20300\%20millones \%20de,en\%20el\%20lugar\%2 0de\%20trabajo.

OPS. Estado de salud de la población salud mental en las américas.

https://www.paho.org/saluden-las-americas-

2017/?post_t_es=la-saludmental-en-la-region-de-lasamericas\&lang=es.

Cabeza Ángel., Guerrero Jairo., García Mario., Lavandero Geovanny., (2018). Niveles de ansiedad entre estudiantes que inician y culminan la carrera en actividad física. Revista. Cubana de Investigaciones Biomédicas. 32 
http://scielo.sld.cu/scielo.php?

script=sci_arttext\&pid=s0864-

03002018000200007

Ramírez Jairo., Quintero Diego., Córdoba Carmen., Ceballos Francisco., Córdoba Franklin., (2020). Consecuencias de la pandemia covid 19 en la salud mental asociadas al aislamiento social. Revista Colombiana de Anestesiología $48 \quad(4)$. https://preprints.scielo.org/ind ex.php/scielo/preprint/downlo $\mathrm{ad} / 303 / 358 / 344$

Rodríguez José., González Agustín., Linares Vitoriano., Burón Raúl., López Luis., Oblitas Luis., (2008). Ansiedad, depresión y salud. Revista. Suma Psicológica. 15 (1). https://www.redalyc.org/pdf/1 342/134212604002.pdf

Sierra Juan., Ortega Virgilio., Zubeidat Inhab., (2003). Ansiedad, angustia y estrés: tres conceptos a diferenciar. Revista. Mal-estar E Subjetividade. 3 (1). https://www.redalyc.org/pdf/2 71/27130102.pdf

Truffino Javier., (2010). Resiliencia: una aproximación al concepto. Revista. Psiquiatría y salud mental. 3 (4). https://doi.org/doi:

10.1016/j.rpsm.2010.09.003 